\title{
Analysis Model in the Cloud Optimization Consumption in Pricing the Internet Bandwidth
}

\author{
Indrawati, Fitri Maya Puspita, Sri Erlita, Inosensius Nadeak \\ Department of Mathematics, Faculty of Mathematics and Natural Sciences, Sriwijaya University, Indonesia
}

\begin{tabular}{l} 
Article Info \\
\hline Article history: \\
Received May 2, 2018 \\
Revised Jul 2, 2018 \\
Accepted Jul 21, 2018 \\
\hline
\end{tabular}

\section{Keyword:}

Cloud computing

Internet pricing

LINGO

\begin{abstract}
The problem of internet pricing is a problem that is often a major problem in optimization. In this study, the internet pricing scheme focuses on optimizing the use of bandwidth consumption. This research utilizes modification of cloud model in finding optimal solution in network. Cloud computing is computational model which is like network, server, storage and service that is utilizing internet connection. As ISP's Internet service provider requires appropriate pricing schemes in order to maximize revenue and provide quality of service (Quality on Service) or QoS so as to satisfy internet users or users. The model used will be completed with the help of LINGO software program to get optimal solution and accurate result. Based on the optimal solution obtained from the modification of the cloud model can be utilized ISP to maximize revenue and provide services in accordance with needs and requests.
\end{abstract}

Copyright (C) 2018 Institute of Advanced Engineering and Science. All rights reserved.

\section{Corresponding Author:}

Fitri Maya Puspita,

Department of Mathematics, Faculty of Mathematics and Natural Sciences, Sriwijaya University,

Jln. Raya Palembang-Prabumulih KM 32 Inderalaya, Ogan Ilir, Indonesia.

Email: fitrimayapuspita@unsri.ac.id

\section{INTRODUCTION}

Internet modernization takes an active role in human activities and life. For some people, internet is already a part of their lives. The Internet is a collection of computer networks that are connected to each other. In the internetaccess it is required a standard protocol such as (TCP) Protocol Transmission Control or (IP) Internet Protocol duty to provide addresses and identities on each computer in order to avoid errors in sending data [1]. In today's modern era, internet users have met all the low, middle, and upper class. Internet usage also do not care the age, from young to old. The growth of users involving all circles and ages is influenced by the life style that follows the development of the era that impact on the pattern of life. In today's terms, the Internet can be said to be a library in which there are kinds of information elements such as text, video, graphics, and sound, will have a lot of very complete information [2].

The more Internet users, the greater the demand for quality. As an Internet service provider, the Internet Service Provider (ISP) should provide better quality and better quality of service to users in achieving the best quality of information at a cost that is efficient. Therefore ISPs are required to provide proper Internet cost planning mechanisms to benefit ISPs as service providers and users as internet users [3].

Increasing the number of internet users is certainly directly proportional to the increase in the amount of bandwidth consumption. The amount of bandwidth consumption is certainly related to the cost. Bandwidth is a quantity that shows the amount of data that can pass in a network connection. Common internet pricing schemes are flat rate, usage-based and two-part tariff. Based on previous research on nonlinear wireless internet pricing schemes compiled by [4], internet pricing schemes on multiple QoS for single link [5], wireless internet pricing schemes are usually associated with QoS bandwidth attributes, bit error rate (BER), end-to-end delay [6]. Optimal internet pricing is required by considering network services. Internet pricing schemes arethe problems and require the right solutions to benefit ISPs and users. 
Research conducted by [7] stated that one of the strategies that the ISP can do to minimize costs and maximize profits is bundle pricing. Bundle pricing can be interpreted as a marketing practice where two or more different products are put together in one package. In addition to bundle pricing, According to [8], utility functions relate to the level of satisfaction consumers get for the consumption of information services that can maximize profits to achieve certain goals.

In previous research done by [9], [10] the pricing scheme to allocate QoS and maximize ISP revenuehade been also discussed. The pricing scheme is done to make resource efficiency to find optimization problem solutions using bottlenecks [3] also conduct research on QoS allocations involving a single route from the source destination.

Every Internet user must have a collection of files stored in electronic-files. Some files may be obtained from e-mail, attachments, downloads, and more. In general, documents stored in various hard media, such as hard disk, flashdisk, PC and laptop for instance, StaaS [11]. In file storage, it has some constraints such as file loss, lost storage media, exposed viruses and so on. Therefore, it would be nice if the storage of data or files built and stored in one place that is electronic files. In addition to provide easy access that can be done anytime and anywhere, electronic file storage also helps in terms of data loss.

Problems about the network much discussed in the world of computers and business world. Network problem is mostly done in internet pricing optimization problem. Cloud computing problems become one of the problems in the network that is warmly discussed today [12]. Cloud computing [13] is one of the computing model that can be accessed anywhere and anytime. Cloud computing is an on-demand service access to a collection of computing resources such as networks, servers, storage, applications such as secure voting system using criptography [14] and services. The main concern about cloud computing is reliability issues in providing computing needs as required by users such as performing the process together, sending and receiving files together. This is because the cloud system will serve as a physical server that will drive multiple virtual servers. The last few years, the development of cloud computing was once discussed by [15] concerning useful parallel computing. To divide the task into several more computing and/or storage resources, which create a large scale system and send back the results to the user. Problems about scheduling tasks in the environment of cloud computing have also been discussed by [16], [17]. Research by [18] and [19] have also formulated task scheduling problems that are run using multiprocess systems so that the length of the schedule can be minimized, and employing genetic algorithms for optimization other than that [20] discusses hemorrhage particle optimization (PSO) based on cloud resource application schedules that take into account both computational costs and data transmission costs.

This study aims to study and analyze schemes for cloud networks and to formulate new dynamic model plans and can work under wireless network clouds. This study focuses on optimizing the use of bandwidth in which the model of the cloud will be completed using the optimization method. Cloud model itself will be simplified into the mathematical model first by determining the purpose function and function constraints. The advantages of this research include research on the cloud model is still new and has not been much discussed. Besides that, this research can be appreciated and useful for internet service provider (ISP) who can apply this method so as to benefit businessman. This study also has advantages which the case raised from this study focuses on the consumption of bandwidth which is the thing that remains a hot issue in the network world. With this research, the expextation to use the data bandwidth can be optimized. Research on optimization of cloud computing model has also rarely been explored so this research becomes the main contribution of this research.

\section{RESEARCH METHOD}

In this research, the calculation will be completed by using the optimization solution in the form of Mixed Integer Linear Programming (MILP) by simulating the form of optimization model using LINGO software. The study uses secondary data of internet bandwidth consumption which is the traffic mail data obtained from the local server in Palembang which then the data will be substituted into the appropriate paramater on the existing model.

\section{RESULTS AND ANALYSIS}

In this study the optimization model used is basedare as follows

$$
\text { Minimize } \sum_{i \in N} \sum_{j \in F_{i}} \alpha_{i j} \cdot p b_{i j}+\left(y_{i j}-\alpha_{i j}\right) \cdot p b_{i j}
$$




$$
\begin{aligned}
& \text { Subject to } \sum_{k \in M} d_{k} \cdot x_{i j k} \leq \operatorname{cap}_{i j} \cdot y_{i j} \quad \forall i \in N, \forall j \in F_{i} \\
& \sum_{i \in N} \sum_{j \in F_{i}} x_{i j k}=1 \quad \forall k \in M \\
& \sum_{j \in F_{i}} y_{i j} \leq 1 \quad \forall i \in N \\
& \begin{array}{l}
\alpha_{i j} \leq y_{i j} \\
x_{i j k} \in\{0,1\}, \quad \forall i \ni N, \forall j \in F_{i} \\
y_{i j} \in\{0,1\},
\end{array}
\end{aligned}
$$

The objective function is shown in (1) and the constraint functions are shown in (2), (3), (4), and (5). Constraint (2) is used to avoid the possibility of a solution that will exceed the capacity set. Constraint (3) ensures that the server assigns the usage properly. Constraint (4) shows that only one frequency $j$ on $i$ will be selected. Constraint (5) is used to bind the decision variable $y_{i j}$ with $\alpha_{i j}$ to the objective function. After defining the existing model, the next definition of each parameter and the variables used in the model in Table 1 and 2 will be explained.

Table 1. Parameters for Each Model

\begin{tabular}{cl}
\hline Parameter & Definition \\
\hline$p b_{i j}$ & Busy bandwidth to run server $i$ at frequency $j$ \\
$p i_{i j}$ & Active-idle bandwidth to run server $i$ at frequency $j$ \\
$d_{k}$ & Workload demand of application \\
$c a p_{i j}$ & Max performance or capacity \\
\hline
\end{tabular}

Table 2. Variables for Each Model

\begin{tabular}{cl}
\hline Variable & Definition \\
\hline$\alpha_{i j}$ & Utilization of server $i$ running at frequency $j$ \\
$y_{i j}$ & Binary variable \\
$x_{i j k}$ & Binary variable \\
\hline
\end{tabular}

After defining each parameter and variable used, in Table 3 we will show the data used. The data used in this research is secondary data obtained from one of the local server. The data used is data traffic mail that is divided into two sessions, namely when the use of the server in busy times and the use of idle servers but remain active.

In this study there are two different cases based on the usefulness of each model. In case I is a general model working on the server while in case II is used to balance the use of the server so it can be used when the workload exceeds the capacity because in case I cannot be used for conditions when workload exceeds capacity, ie

a. Case I

In case I the model form will follow the main model form, so there is no significant difference. In other words, the objective function in case I with the main model will be the same so the constraint function will remain the same.

b. Case II

In case II that distinguish is in case II this condition used will be different that is $x_{i j k} \in[0,1]$

In addition, the function constraints (2), and (3) to be changed with the function constraints (6), and (7) as follows

$$
\begin{aligned}
& \sum_{i \in N} \sum_{j \in F_{i}} \operatorname{cap}_{i j} \cdot x_{i j k} \geq d_{k} \quad \forall k \in M \\
& \sum_{k \in M} x_{i j k} \leq y_{i j} \quad \forall i \in N, \forall j \in F_{i}
\end{aligned}
$$


In this study, there are also several conditions that follow two previous cases. There are 4 conditions for each case. Conditions are distinguished by differences in the selection of frequency values for each server. Some of these conditions are made in order to adjust to any possibilities that exist so that the model can still be used.

a. Condition 1

In condition 1, selected frequency as follows

$F_{i}= \begin{cases}\{1,2\}, & \text { for } i=1 \\ \{3,4\}, & \text { for } i=2\end{cases}$

b. Condition 2

In condition 2 selected frequency for each server $\mathrm{i}$ is the same, that is

$F_{i}=\{1,2\}, \quad$ for $i=1,2$

c. Condition 3

In condition 3, selected frequency as follows

$F_{i}= \begin{cases}\{1,2\}, & \text { for } i=1 \\ \{3,4,5\}, & \text { for } i=2\end{cases}$

d. Condition 4

In condition 4, selected frequency as follows

$F_{i}= \begin{cases}\{1,2,3\}, & \text { for } i=1 \\ \{3,4\}, & \text { for } i=2\end{cases}$

Table 3. Value of Each Parameter

\begin{tabular}{cc}
\hline Parameters & Value \\
\hline$p b_{11}$ & 5334.928178 \\
$p b_{12}$ & 9944.332127 \\
$p b_{13}$ & 8802.4507 \\
$p b_{21}$ & 9320.495228 \\
$p b_{22}$ & 10837.64875 \\
$p b_{23}$ & 10309.12084 \\
$p b_{24}$ & 10604.62512 \\
$p b_{25}$ & 9093.060161 \\
$p i_{11}$ & 7882.625121 \\
$p i_{12}$ & 11630.88486 \\
$p i_{13}$ & 12563.42076 \\
$p i_{21}$ & 12904.85285 \\
Parameters & Value \\
$p i_{22}$ & 11175.84852 \\
$p i_{23}$ & 13193.51597 \\
$p i_{24}$ & 12645.80803 \\
$p i_{25}$ & 11929.0641 \\
$d_{1}$ & 1149 \\
$d_{2}$ & 1290 \\
$c a p_{11}$ & 1553 \\
$c a p_{12}$ & 1607 \\
$c a p_{13}$ & 1068 \\
$c a p_{21}$ & 1724 \\
$c a p_{22}$ & 1811 \\
$c a p_{23}$ & 1766 \\
$c a p_{24}$ & 1819 \\
$c a p_{25}$ & 1937 \\
\hline & \\
\hline & \\
&
\end{tabular}

After determining the existing case and the various conditions used, then the model is solved by using LINGO as an application to solve the optimization problem. Table 4 and Table 5 show the results of the LINGO solution for each of the existing cases, as follows: 
Table 4. Solver Status for Case 1 on Each Condition

\begin{tabular}{ccccc}
\hline \multirow{2}{*}{ Solver Status } & Condition I & Cindition II & Condition III & Condition IV \\
\hline Model Class & MILP & MILP & MILP & MILP \\
State & Global Optimal & Global Optimal & Global Optimal & $\begin{array}{c}\text { Global Optimal } \\
\text { Objective }\end{array}$ \\
Infeasibility & 0 & 14655.42 & 14427.99 & 5334.928 \\
Iteration & 0 & 0 & 0 & 0 \\
& & 0 & 0 & 0 \\
Solver Type & Branch and Bound & Branch and Bound & Branch and Bound & Branch and Bound \\
Best Objective & 15644.05 & 14655.42 & 14427.99 & 5334.928 \\
Steps & 0 & 0 & 0 & 0 \\
Update & 2 & 2 & 2 & 2 \\
Interval & 28 & 28 & 31 & 31 \\
GMU (K) & 1 & 0 & 0 & 0 \\
ER (Sec) & & & & 2 \\
\hline
\end{tabular}

In the case I for the four conditions, the greatest objective solution was found in condition I with the optimal solution obtained was 15644.05 obtained through 21 iterations with no infeasibility. Generated Memory Used (GMU) shows the amount of memory allocation used is $28 \mathrm{~K}$ and Elapsed Runtime (ER) describes the total time spent to generate and complete the model. ER for condition I is 0 seconds.

Table 5. Solver Status for Case 2 on Each Condition

\begin{tabular}{|c|c|c|c|c|}
\hline \multirow{2}{*}{ Solver Status } & \multicolumn{4}{|c|}{ Case II } \\
\hline & Condition I & Condition II & Condition III & Condition IV \\
\hline Model Class & MILP & MILP & MILP & MILP \\
\hline State & Global Optimal & Global Optimal & Global Optimal & Global Optimal \\
\hline Objective & 15644.05 & 14655.42 & 14427.99 & 17264.99 \\
\hline Infeasibility & 0 & 0 & 0 & 0 \\
\hline Iteration & 21 & 22 & 26 & 24 \\
\hline \multicolumn{5}{|c|}{ Extended Solver Status } \\
\hline Solver Type & Branch and Bound & Branch and Bound & Branch and Bound & Branch and Bound \\
\hline Best Objective & 15644.05 & 14655.42 & 14427.99 & 17264.99 \\
\hline Steps & 0 & 0 & 0 & 0 \\
\hline $\begin{array}{l}\text { Update } \\
\text { Interval }\end{array}$ & 2 & 2 & 2 & 2 \\
\hline GMU (K) & 30 & 30 & 33 & 33 \\
\hline $\mathrm{ER}(\mathrm{Sec})$ & 0 & 0 & 0 & 0 \\
\hline
\end{tabular}

In the second case for all four conditions, the greatest objective solution was obtained in condition IV with the optimal solution obtained was 17264.99 obtained through 24 iterations with no infeasibility. Generated Memory Used (GMU) shows the amount of memory allocation used for 33K and Elapsed Runtime (ER) describes the total time used to generate and complete the model. ER for condition IV is 0 seconds. For the solution of each variable used in the model for each case and for each condition is shown in Table 6.

Table 6. Solution for Each Variables

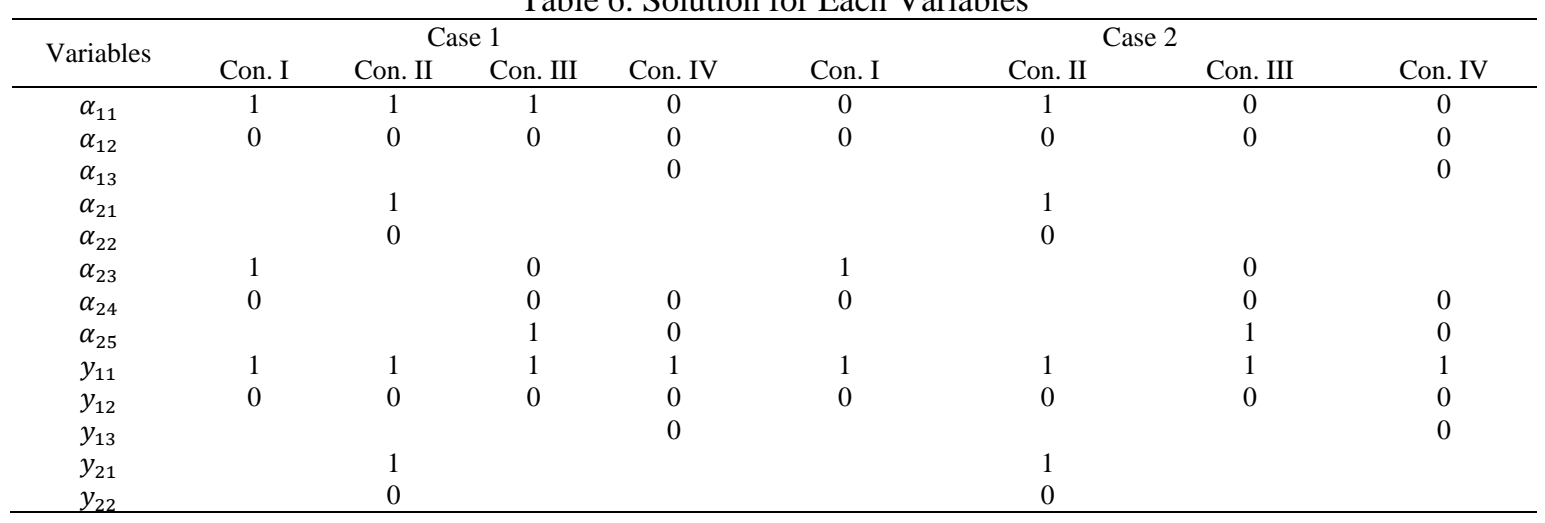


Table 6. Solution for Each Variables

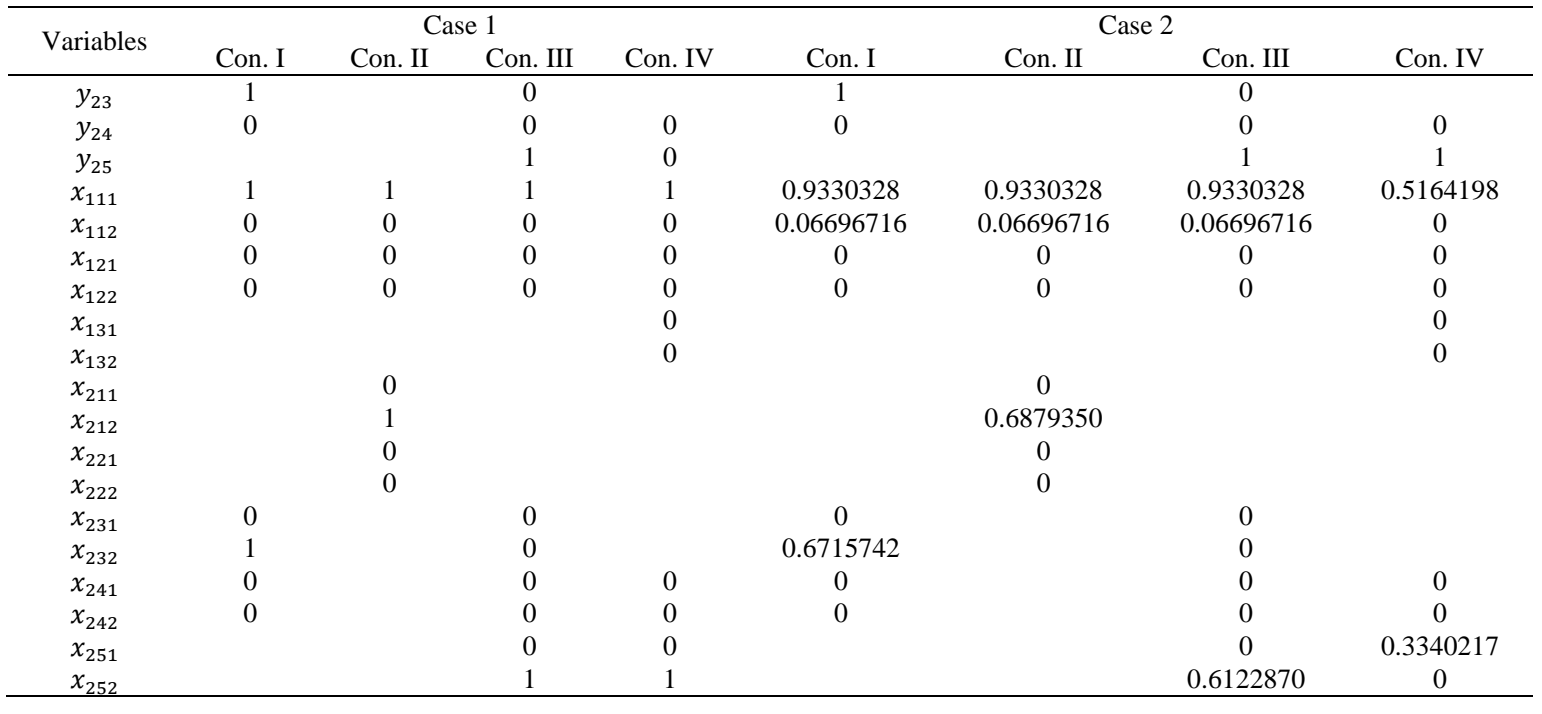

The variable solutions show us that for some parts, the values are 0 s or 1 s depending on the conditions as forms of mixed integer linear programming problem. The solutions only work for Case 1 problem only. Since for Case 2, some variable values violate the integer constraints.So, the Case 1 where the model form will follow the main model form will be the best solution that ISP can consider in gaining the profit by utilizing the cloud optimization-pricing scheme.

\section{CONCLUSION}

In this research, there are 2 different cases that have 4 conditions respectively. From the solution using LINGO it was found that the results for each condition differ from each other but all are in the form of Mixed Integer Linear Programming (MILP) with global solution.

\section{ACKNOWLEDGEMENTS}

The research leading to this study was financially supported by Sriwijaya University for support through Competitive Leading Grant in 2017.

\section{REFERENCES}

[1] Y. Maryono and B.P. Istiana, "Teknologi Informasi dan Komunikasi", A. B. Darmadi, Ed., 2008.

[2] L. Sidharta, "Internet Informasi Bebas Hambatan", Jakarta, Elex Media Komputindo, 1996.

[3] J. Byun and S. Chatterjee, "A strategic pricing for quality of service (QoS) network business", in Proceedings of the Tenth Americas Conference on Information Systems, New York, 2004.

[4] E. Wallenius and T. Hämäläinen, "Pricing Model for 3G/4G Networks", in 13th IEEE International Symposium on Personal, Indoor, and Mobile Radio Communications, 2002.

[5] F.M. Puspita, K. Seman, B.M. Taib, and Z. Shafii, "Improved Models of Internet Charging Scheme of Single Bottleneck Link in Multi QoS Networks", Journal of Applied Sciences, vol. 13, pp. 572-579, 2013.

[6] Indrawati, Irmeilyana, F.M. Puspita, and O. Sanjaya, "Internet pricing on bandwidth function diminished with increasing bandwidth utility function", TELKOMNIKA, vol. 13, pp. 299-304, 2015.

[7] S. Viswanathan and G. Anandalingam, "Pricing strategies for information goods", pp. 257-274, 2005.

[8] X. Wang and H. Schulzrinne, "Pricing network resources for adaptive applications in a differentiated services network", in Proceedings of IEEE INFOCOM 2001, 2001.

[9] W. Yang, H.L. Owen, and D.M. Blough, "A Comparison of Auction and Flat Pricing for Differentiated Service Networks", in Proceedings of the IEEE International Conference on Communications, 2004.

[10] W. Yang, H.L. Owen, and D.M. Blough, "Determining Differentiated Services Network Pricing Through Auctions", in Networking-ICN 2005, 4th International Conference on Networking April 2005, 2005. 
[11] S. Roy, P.K. Pattnaik, and R. Mall, "A cognitive Approach for Evaluating the Usability of Storage as a Service in Cloud Computing Environment", International Journal of Electrical and Computer Engineering (IJECE), vol. 6, pp. 759-769, 2016.

[12] K.K. Chennam and M.A. Lakshmi, "Cloud Security in Crypt Database Server Using Fine Grained Access Control", International Journal of Electrical and Computer Engineering (IJECE), vol. 6, pp. 915-924, 2016.

[13] T. Sasidhar, V. Havisha, S. Koushik, M. Deep, and V.K. Reddy, "Load Balancing Techniques for Efficient Traffic Management in Cloud Environment", International Journal of Electrical and Computer Engineering (IJECE), vol. 6, pp. 963-973, 2016.

[14] M. Ranjan, A.H. Mondal, and M. Saikia, "A Cloud Based Secure Voting System using Homomorphic Encryption for Android Platform", International Journal of Electrical and Computer Engineering (IJECE), vol. 6, pp. 29943000, 2016.

[15] V. Kumar, A. Grama, A. Gupta, and G. Karypis, "Introduction to parallel computing", vol. 110, 1994.

[16] D. Agrawal, S. Das, and A.E. Abbadi, "Big data and cloud computing: current state and future opportunities", in 14th International Conference on Extending Database Technology, ACM, 2011.

[17] V. Petrucci, O. Loques, and D. Mosse, "A Dynamic Optimization Model for Power and Performance Management of Virtualized Clusters", in Proceeding e-Energy '10 Proceedings of the 1st International Conference on EnergyEfficient Computing and Networking Passau, Germany, 2010.

[18] E.S.H. Hou, N. Ansari, and H. Ren, "A genetic algorithm for multiprocessor scheduling, Parallel and Distributed Systems," International Journal of Grid and Distributed Computing, vol. 7, 2014.

[19] P. Varalakshmi, A. Ramaswamy, A. Balasubramanian, and P. Vijaykumar, "An Optimal Workflow Based Scheduling and Resource Allocation in Cloud", in Computing and Communications, 2011, pp. 411-420.

[20] S. Pandey, L. Wu, S. Guru, and R. Buyya, "A Particle Swarm Optimization-Based Heuristic for Scheduling Workflow Applications in Cloud".

\section{BIOGRAPHIES OF AUTHORS}

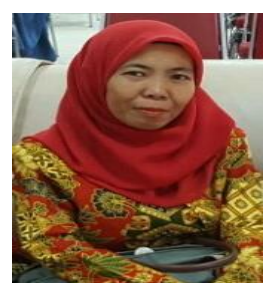

Indrawatiher S.Si degree in Mathematics from Sriwijaya University, South Sumatera, Indonesia in 1996. Then she received her M.Si in Actuarial Science in 2004. She has been a Mathematics Department member at Faculty mathematics and Natural Sciences Sriwijaya University South Sumatera Indonesia since 1998. Her research interests include optimization, actuarial science and insurance problems.

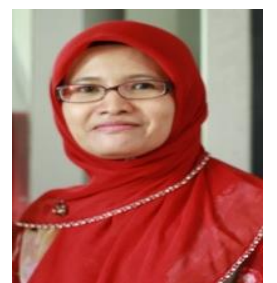

Fitri Maya Puspita received her S.Si degree in Mathematics from Sriwijaya University, South Sumatera, Indonesia in 1997. Then she received her M.Sc in Mathematics from Curtin University of Technology (CUT) Western Australia in 2004. She reveived his Ph.D in Science and Technology in 2015 from Universiti Sains Islam Malaysia. She has been a Mathematics Department member at Faculty mathematics and Natural Sciences Sriwijaya University South Sumatera Indonesia since 1998. Her research interests include optimization and its applications such as vehicle routing problems and QoS pricing and charging in third generation internet.

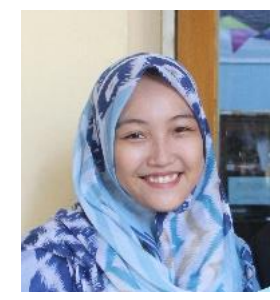

Sri Erlita currently is an undergraduate student at Mathematics Department, Faculty of Mathematics and Natural Sciences, Sriwijaya University. She is currently on final stage of her thesis submission. Her topic interest includes Optimization and its application on pricing of information service in cloud environment.

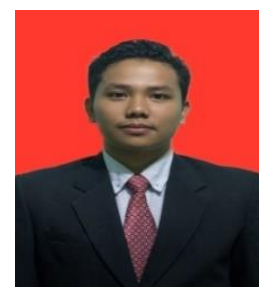

Inosensius Nadeak currently is an undergraduate student at Mathematics Department, Faculty of Mathematics and Natural Sciences, Sriwijaya University. He is currently on final stage of her thesis submission. His topic interest includes Optimization and its application on pricing of information service e in cloud environment. 